\title{
Hydrodynamic deformation reveals two coupled modes/time scales of red blood cell relaxation
}

\author{
Susanne Braunmüller, ${ }^{a}$ Lothar Schmid, ${ }^{a}$ Erich Sackmann ${ }^{c}$ and Thomas Franke ${ }^{* a b}$ \\ Received 29th June 2012, Accepted 17th August 2012 \\ DOI: $10.1039 / \mathrm{c} 2 \mathrm{sm} 26513 \mathrm{c}$
}

We study the mechanical relaxation behavior of human red blood cells by observing the time evolution of shape change of cells flowing through microchannels with abrupt constrictions. We observe two types of relaxation processes. In the first fast process $\left(\tau_{1} \sim 200 \mathrm{~ms}\right)$ the initially parachute shaped cells relax into cup-shaped cells (stomatocytes). These cells relax and reorient in a second relaxation process with a response time of $\tau_{1 / 2} \sim 10 \mathrm{~s}$ into the equilibrium discoid shapes. The values for the relaxation times of single red blood cells in the population scatter significantly within the cell population between $0.11 \mathrm{~s}<\tau_{1}<0.52 \mathrm{~s}$ and $9 \mathrm{~s}<\tau_{1 / 2}<49 \mathrm{~s}$, respectively. However, when plotting $\tau_{1 / 2}$ against $\tau_{1}$, we find a linear relationship between the two timescales and are able to relate both to the elastic properties of the spectrin cytoskeleton underlying the red cell's plasma membrane. Adenosine Triphosphate (ATP) enhances dissociation of spectrin filaments resulting in a reduced shear modulus. We modify the cytoskeleton connectivity by depletion and repletion of ATP and study the effect on relaxation. Both the linear relationship of timescales as well as the ATP dependence can be understood by theoretical models.

\section{Introduction}

During their transport through the capillaries of blood vessels, erythrocytes are repeatedly strongly deformed undergoing transitions from parachute to discoid shapes. A high degree of elastic deformability of the cells is essential to enable them to move during their lifetime of $\sim 120$ days through the narrow capillaries exhibiting diameters smaller than those of the red cells. The astonishing softness of red blood cells in microcirculation and the enormous reversible shape deformability are based on the large surface to volume ratio and on the mechanical properties of the lipid bilayer and the underlying cytoskeleton. While the fluid lipid/protein bilayer of the composite cell envelope exhibits only bending resistance (characterized by the bending modulus $\kappa$ ), the deformation of the cytoskeleton is controlled by shear elasticity (characterized by the shear modulus $\mu$ ) and actively regulated by ATP. ${ }^{1}$

Both moduli have been measured with different techniques including deformation in high frequency electric fields, ${ }^{2,3}$ micropipette manipulation, ${ }^{4}$ optical tweezers, $\mathrm{AFM}^{2,4-7}$ and analysis of membrane fluctuation. ${ }^{8,9}$ Some of these measurements show the relaxation of the deformed red cell shape into its equilibrium shape which occurs after switching off the external

${ }^{a}$ Microfluidics Group, EP1, Universität Augsburg, D-86159 Augsburg, Germany.E-mail: Thomas.Franke@physik.uni-augsburg.de

${ }^{b}$ Center for NanoScience (CeNS), Ludwig-Maximilians-Universität München, D-80539 München, Germany

'Physics Department, Institute for Biophysics E22, Technische Universitaet München, D-85748 Garching, Germany forces. The dissipating terms opposing the elastic restoring stresses during the relaxation can be either determined by the membrane viscosity $\eta_{\mathrm{m}}$ of the cell envelope or the bulk viscosity of the cytoplasm $\eta$. This balance yields characteristic time scales of shape relaxation. Most authors report a fast relaxation process characterized by relaxation times of 100-300 ms. Only recently, a significantly longer time scale of several seconds was reported by Fischer. He found a red cell rotational relaxation with conserved outer cell shape and explained it in terms of a shape memory. ${ }^{10}$ However, both types of relaxation have never been observed together.

We have observed for the first time both modes of shape and rotational relaxation simultaneously in one single continuous experiment by analyzing the relaxation of parachute shaped red cells. This allows us to consecutively measure both timescales of individual cells and to establish a linear relationship between the fast and the slow relaxation times. The rotational relaxation time increases (decreases) with increasing (decreasing) relaxation time of the shape deformation. We conclude that both relaxation processes depend on the mechanical properties of the cytoskeleton.

The cytoskeleton consists of semiflexible spectrin filaments that form a nearly triangular network with the vertices interconnected by actin oligomers, and is locally coupled with integral proteins at the cytoplasmic face of the lipid/protein bilayer. The association is mediated by two types of linker molecules. Ankyrin couples the interconnected head-to-tail connections of the spectrin hetero-dimers to the intracellular domain of the protein band III. The other linker protein is band IV.1 and 
couples the actin oligomer that connects the spectrin tetramer of the network to the membrane spanning receptor glycophorin. The mechanical properties of the cytoskeleton network are controlled by the density of defects of the network and the lateral density of cytoskeleton-membrane linkages and are the result of a dynamical process of the cytoskeletal protein rearrangement by dissociation and re-association. ${ }^{11}$ ATP promotes spectrin-actin dissociation and induces transient cytoskeletal dissociations that account for a reduced shear elasticity. ${ }^{12-15}$

By analyzing the effect of ATP depletion on the relaxation we demonstrate that relaxation is affected by variations in cytoskeleton network shear elasticity. Depletion of ATP results in a substantial decrease of the slow rotational relaxation time and the effect is reversible after adding ATP again.

Evidence for regulation of red blood cell shape and mechanical function by ATP-induced protein skeleton modification has been reported by several other groups. ${ }^{\mathbf{8}, 16-19}$ In this process ATPconsuming protein kinases regulate the phosphorylation state of the membrane protein IV.1 and the dissociation of spectrin from protein IV.1. ${ }^{12,20}$ Hence, depletion of ATP results in a reduced enzyme activity and in the stiffening of the red cell envelope by slowing down of the dissociation of the cytoskeleton-membrane coupling. On the contrary, red blood cells release ATP upon deformation which is known to help blood pressure regulation. ${ }^{21-23}$ External shear forces induce spectrin tetramers to break into dimers ${ }^{24}$ or unfolding of spectrin subdomains may occur ${ }^{25}$ both of which are accompanied by network softening., ${ }^{9,26,27}$ Only recently it was shown by Forsyth et al. that ATP release correlates also with bulk shear thinning of suspended red cells. ${ }^{21}$ This delicate mechano-biomolecular interplay is essential for blood function and regulation.

Several diseases are related to failure of cytoskeletal proteinnetwork connectivity and biochemical function. ${ }^{28-30}$ Decreased RBC deformability and increased cell fragility in hereditary spherocytosis are caused by a reduced number of vertical linkages between bilayer and skeleton whereas in hereditary elliptocytosis horizontal linkages in the skeletal network are defective. ${ }^{31,32}$ Plasmodium infected erythrocytes in malaria lose their native deformability due to reduction in intracellular ATP levels. ${ }^{1,33}$

We deform single red cells by hydrodynamic shear forces using a microfluidic setup ${ }^{34}$ in which the cells are transiently deformed into a parachute-like shape. ${ }^{35,36}$ They then enter an abrupt widening of the channel in which the flow velocity is drastically reduced and relax into the discoid equilibrium shape.

\section{Preparation/setup}

Blood was freshly drawn from healthy donors and anti-coagulated with ethylendiaminetetraacetate (EDTA). Subsequently, the whole blood was washed three times in isoosmotic phosphate buffered saline (PBS) at $\mathrm{pH}$ 7.4. RBC with decreased ATP levels were prepared by metabolic depletion in PBS at $4{ }^{\circ} \mathrm{C}$ for $48 \mathrm{~h}$ and 72 h. ${ }^{37}$ To replete red cells with ATP metabolically depleted cells were incubated with PBS containing 2 mM ATP (Sigma Aldrich) for 1 hour.

For the experiment the cells were resuspended in PBS containing $10 \mathrm{mg} \mathrm{ml}^{-1}$ bovine serum albumin (BSA) at a final concentration of $0.1 \%$ hematocrit and transferred into a sterile syringe that was connected to the microfluidic channel and mounted on an inverted phase contrast microscope (Zeiss, Axiovert 200M). The cell flow rate was controlled by adjusting the hydrostatic pressure between the inlet and outlet of the fluidic device. Microchannels were fabricated using standard polydimethylsiloxane (PDMS) soft-lithography. ${ }^{38}$

\section{Experimental}

Freely suspended red blood cells are injected into a microfluidic PDMS device and passed through a hyperbolically narrowing constriction of the microchannel of $2.5 \mu \mathrm{m}$ width at the orifice, a constant height of $8 \mu \mathrm{m}$ and with a total length of $\sim 500 \mu \mathrm{m}$. Typical cell flow velocities are $\sim 300 \mu \mathrm{m} \mathrm{s}^{-1}$. After passing the constriction, cells enter into a much wider reservoir with a channel width of a factor $\sim 50$ larger. Consequently, the red cell motion rapidly slows down and stops after about $1 \mathrm{~s}$. In some incidents, flow stopped completely after the cell exited the channel. This flow-stop was caused by subsequent spiculated red cells (echinocytes) clogging the channel. After having slowed down the cells start to relax into the equilibrium discoid shape. Regarding the relaxation behavior of the cells no significant difference could be found between both scenarios of rapid and complete flow-stop. Cells that exit the orifice of the constriction exhibit a parachute-like shape as shown in the top micrographs in Fig. 1. Subsequently, two distinct types of relaxations were observed. In the first step, the parachute shape relaxes and the front and rear curvatures become less convex and concave, respectively (compare image in Fig. 1 after $560 \mathrm{~ms}$ ). In the end of

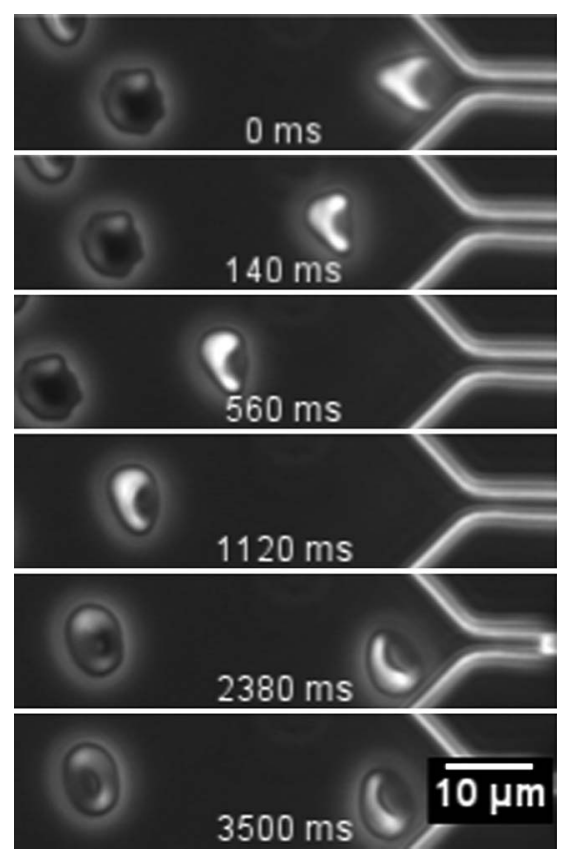

Fig. 1 Observation of both types of relaxations in one single experiment: A red blood cell flowing out of a narrow micro-channel (width $2.5 \mu \mathrm{m}$, height $8 \mu \mathrm{m}$ ). The cell adopts a parachute shape right after leaving the constriction (top micrograph) and relaxes into a stomatocyte (subsequent micrographs up to $\sim 1120 \mathrm{~ms}$ ). In the last two micrographs the red blood cell rotates on a much longer time scale while adopting its discoid equilibrium shape (times between micrographs are as indicated in the images). 
this process the cell adopts the shape of a cup-shaped stomatocyte. ${ }^{39}$ This initial relaxation from a parachute to a stomatocyte happens on a time scale $\tau_{1} \sim 200 \mathrm{~ms}$. However, this fast shape transformation is followed by a much slower shape transformation that is accompanied by a rotational relaxation of the red blood cells. During this second phase the red cell transits from the stomatocyte-shape into its discoid equilibrium shape but concomitantly rotates as a whole as shown in the micrographs and sketches of Fig. 2. The red cells have been observed to rotate in both directions either clockwise or counterclockwise. Both processes of initial fast shape and subsequent slower shaperotational relaxation are temporally clearly separated by approximately one order of magnitude and can be detected successively. The typical time scale $\tau_{1 / 2}$ for the rotational relaxation is several seconds.

We analyze the two phases of relaxation, shape and orientation transformation, by fitting the contour of the red cells using image processing software. For the parachute relaxation the deformation index DI $=l / w$ defined as the ratio of red cell width $w$ to the length $l$ is quantified. From an exponential fit to the plot of the DI against time (see Fig. 3), we obtain the relaxation time $\tau_{1}$ for the parachute-shaped cells. We find a mean value of $\tau_{1}=0.30 \pm 0.20 \mathrm{~s}$.

After this first fast process, a concave dimple at the rear of the red cell can be observed. The dimple is assumed to be a remnant of the concave rear of the parachute shape. Investigation of many cells showed that the cup-shaped cell rotates in a face-on or edgeon configuration while transforming into a discocyte as outlined in Fig. 2. The tilt angle $\varphi$ of the red cell, that is the angle between the plane of the cell and the bottom of the channel as indicated in Fig. 2, decreases with time and eventually approaches a constant value which generally deviates from $\varphi_{\infty}=0$. A tilt angle $\varphi=0$ implies that the red cell is in the face-on configuration with one dimple pointing upwards (central sketch in Fig. 2a). This characteristic dependence was observed for all analyzed red blood cells. The variation of the terminate angle $\varphi_{\infty}$ (saturation) in the different experiments indicates that the tilt is not correlated to the horizontal microchannel alignment, clearly excluding gravitational as well as confinement effects as the cause for rotation.

We parameterized the rotational relaxation by fitting the contour of the cell and the relative position of the dimple. Here, we determined the tilt angle $\varphi$ of the discocyte from the position of the center $R$ of the cell and the apparent position of the dimple center $d$ as depicted in Fig. 2. The tilt angle was derived from the relation $\sin \varphi=d / R$. A typical dependence of $\varphi$ on time is shown in Fig. 3b. The plot displays an approximately linear decrease of $\varphi$ with time as would be expected for constant angular velocity $\omega=\mathrm{d} \varphi / \mathrm{d} t$. For longer times the angle approaches its saturation value $\varphi_{\infty}$. The difference $\Delta \varphi=\varphi_{0}-\varphi_{\infty}$ of the initial angle $\varphi_{0}$ (at $t=0$ ) and the saturation angle varies in the experiments as $0^{\circ}<$ $\Delta \varphi<\pi / 2$. The limitation of the tilt angle to values smaller than $\pi /$ 2 clearly excludes a tumbling motion ${ }^{40,41}$ of red cells because for tumbling the tilt angle increases indefinitely. We define the typical time scale of the rotation $\tau_{1 / 2}=\pi / 4 \omega$ as the time of a rotation of $45^{\circ}$, that is half of the maximum rotation. The experimental values for $\tau_{1 / 2}$ vary in the interval of $9.6 \mathrm{~s}<\tau_{1 / 2}<$ $48.9 \mathrm{~s}$ for normal red blood cells and we find $\tau_{1 / 2}=19.3 \mathrm{~s} \pm 12.2 \mathrm{~s}$.

To gain further insight into the control of the relaxation times by the structure of the spectrin-actin network and its coupling to the lipid/protein bilayer, we studied the influence of ATP depletion on the slow relaxation time $\tau_{1 / 2}$. ATP is known to soften the erythrocyte composite shell which is attributed to the dynamic coupling and decoupling of the spectrin-actin network to the integral proteins of the bilayer,12,14 and to softening (prevent stiffening) of the spectrin links ${ }^{42}$ and is therefore an ideal candidate to control its elastic properties. We altered the cytosolic ATP level by metabolic depletion for up to $72 \mathrm{~h}$ and (a)
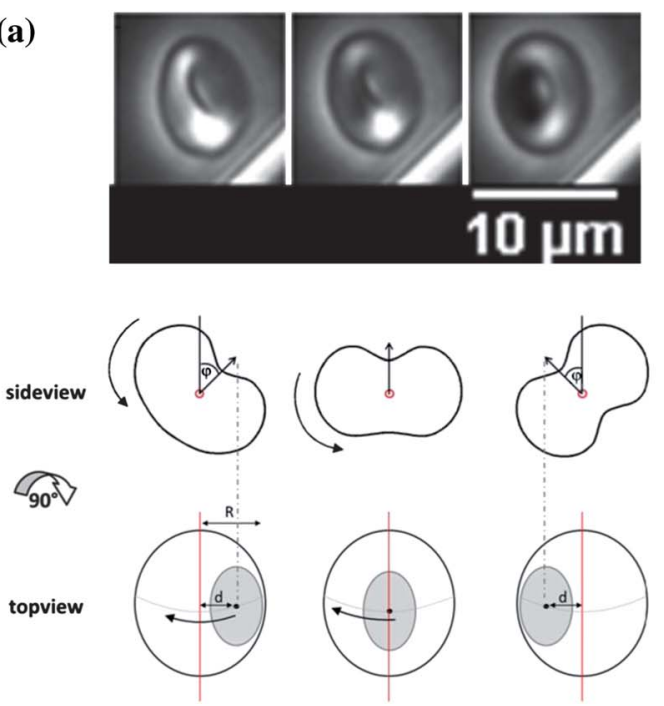

(b)
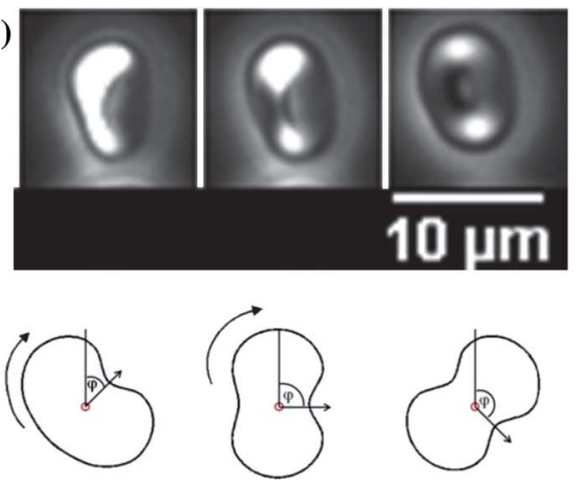

Fig. 2 Close-up phase contrast micrographs show a top view of the slow rotational process (rotational relaxation). The two alternatives of rotational relaxation that depend on the direction of rotation are demonstrated: with face-on (a) and edge-on (b) rotations, respectively. The rotation is accompanied by a shape transformation from the stomatocyte to the discocyte. The schematics above are side view and top view corresponding to the micrographs below to visualize the transformation and the rotation. The tilt angle $\varphi$ of rotation is defined as indicated in the sketch. Note that in (a) the angle $\varphi$ changes its sign from a negative to a positive value. 


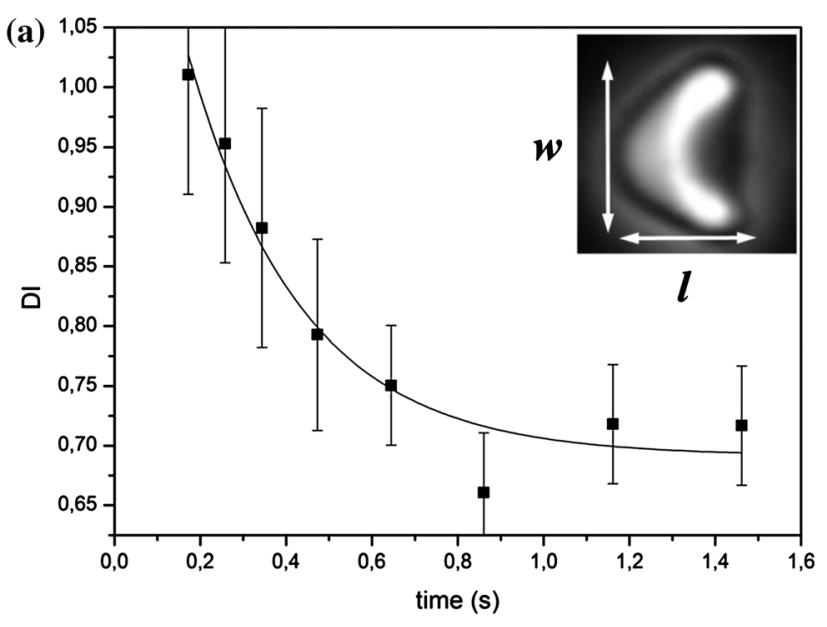

(b)

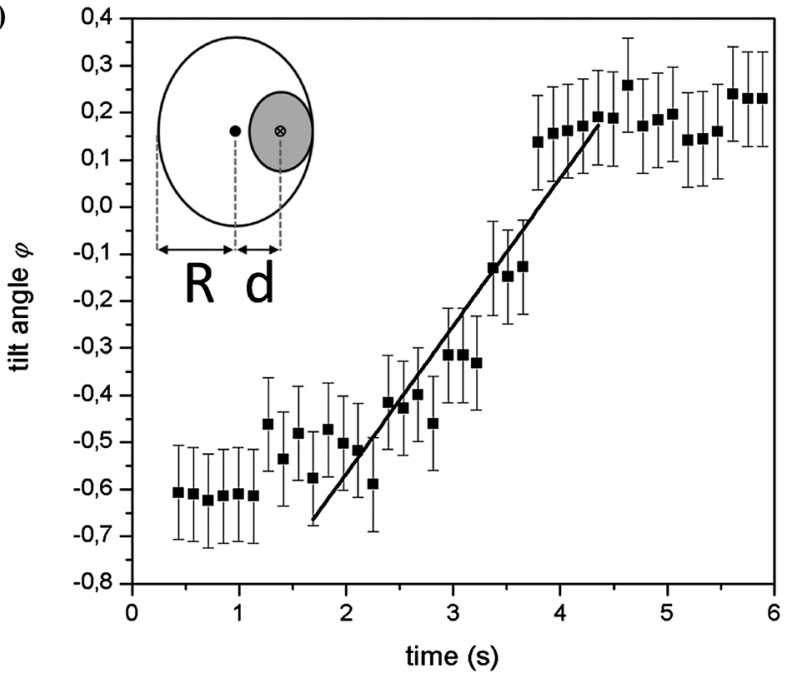

Fig. 3 In (a) and (b) data for a typical single experiment are presented; large error bars are due to resolution (pixel size) of images. (a) Shows the time dependence of the red cell deformation index (DI = cell length/cell width) against time. The continuous line is an exponential fit of the displayed data with decay time $\tau_{1}=0.26 \mathrm{~s} \pm 0.07 \mathrm{~s}$. The inset indicates how the parachute DI $=l / w$ is obtained from the red blood cell micrographs. The mean value for all experiments is $\tau_{1}=0.30 \mathrm{~s} \pm 0.20 \mathrm{~s}$. In (b) the tilt angle $\varphi$ of the discoid red blood cell as defined in Fig. 2 is plotted versus time. Experimentally, the tilt angle $\varphi$ was obtained from the relation $\sin \varphi=d / R$ where $R$ is the radius of the cell and $d$ is the apparent position of the dimple center as depicted in the inset of (b). The plot in (b) exhibits two intervals. In the first interval the tilt angle decreases approximately linearly. In the second interval the tilt angle exhibits a stationary constant value. This type of dependence is typical for all the analyzed experiments. From the slope of the first interval we deduce an angular velocity $\mathrm{d} \varphi / \mathrm{d} t$. From the angular velocity we derive the timescale for a typical mean rotation of $\pi / 4$ by $\tau_{1 / 2}=\frac{\pi}{4}\left(\frac{\mathrm{d} \varphi}{\mathrm{d} t}\right)^{-1}$. In plot (b) this scale yields $\tau_{1 / 2}=6.80 \mathrm{~s}$.

measured the corresponding effect on rotational relaxation time $\tau_{1 / 2}$. After $48 \mathrm{~h}$ about $20 \%$ and after $72 \mathrm{~h}$ about $30 \%$ of RBCs evolved into echinocytes. Echinocytes are crenate erythrocytes with several dozens of spicules. ${ }^{39,43}$ The appearance of this crenate shape corresponds to a $70-80 \%$ reduced ATP level compared to fresh RBCs. ${ }^{17}$ The ATP level in healthy native $\mathrm{RBCs}$ is known to be $\sim 1.3 \mathrm{mM}$.
Only discoid cells and echinocytes I (according to Bessis' nomenclature ${ }^{43}$ ) are taken into account for relaxation measurements. For ATP depleted cells the rotational relaxation time was found to be $\tau_{1 / 2}=7.3 \pm 1.7 \mathrm{~s}$ after 48 hours and $\tau_{1 / 2}=3.6 \pm 2.7 \mathrm{~s}$ after 72 hours after starting the depletion. It is thus significantly smaller compared to native RBCs with $\tau_{1 / 2}=19.3 \pm 12.2 \mathrm{~s}$. The ATP-depletion effects are reversible, since after addition of ATP to depleted cells the reduction of the relaxation time was completely reversed. Treating red cells after $48 \mathrm{~h}$ of ATP depletion with $2 \mathrm{mM}$ ATP, which is about double the concentration of native ATP level, yielded a significant recovery of the rotational relaxation time to a value of $\tau_{1 / 2}=13.4 \pm 5.4 \mathrm{~s}$.

Owing to the large scattering of the measured relaxation times $\tau_{1}$ and $\tau_{1 / 2}$ one would not expect to observe correlations between the two timescales. However, there are different contributions for the large variation in experimental values. Aside from the measurement errors as indicated in Fig. 3 the large scattering is caused by the natural variation of red cell properties within a cell population (see also Fig. 4). To verify if the natural distribution of these properties essentially accounts for the scattering and to correlate the time scales we determined both relaxation times for one and the same red cell consecutively in one experiment as illustrated in Fig. 1. The correlation between $\tau_{1}$ and $\tau_{1 / 2}$ is shown in the plot of Fig. 5 for both ATP depleted and fresh cells. It is seen that a large deformational relaxation time $\tau_{1}$ implies a larger rotational relaxation time $\tau_{1 / 2}$ and vice versa. In fact a linear fit of the data yields a line almost through the origin $\tau_{1}=k \tau_{1 / 2}$, with a constant of proportionality $k=38.5 \pm 4.8$ and a very small value of the axis intersection point. This fact has strong implications for the physical interpretation of the timescales and is discussed in the following.
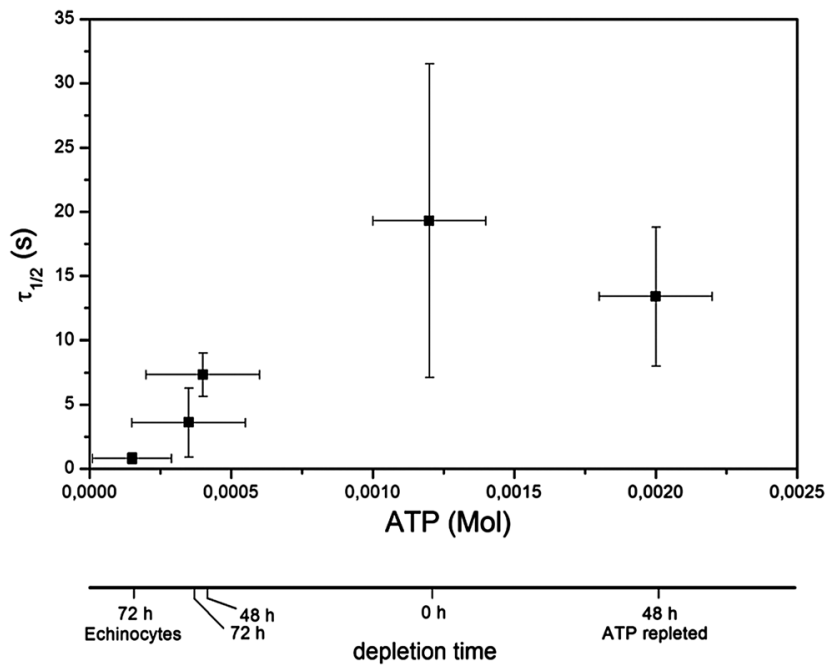

Fig. 4 Dependence of the rotational relaxation time $\tau_{1 / 2}$ of red cells on the ATP level. The ATP level was modified incubating the cell in starving buffer at $4{ }^{\circ} \mathrm{C}$ for a depletion time as shown on the lower axis. ATP levels decrease with time due to metabolic depletion of ATP, as no glucose (or other source of energy) is offered. To test reversibility $2 \mathrm{mM}$ ATP was added after $48 \mathrm{~h}$ depletion time to replete cellular ATP levels. The intracellular ATP concentration was estimated following the procedures described in ref. 1 and 17. Large natural variation of cellular ATP levels ${ }^{17}$ as well as variation of kinase activity ${ }^{56}$ explain the large error bars at high ATP levels (see Discussion). 


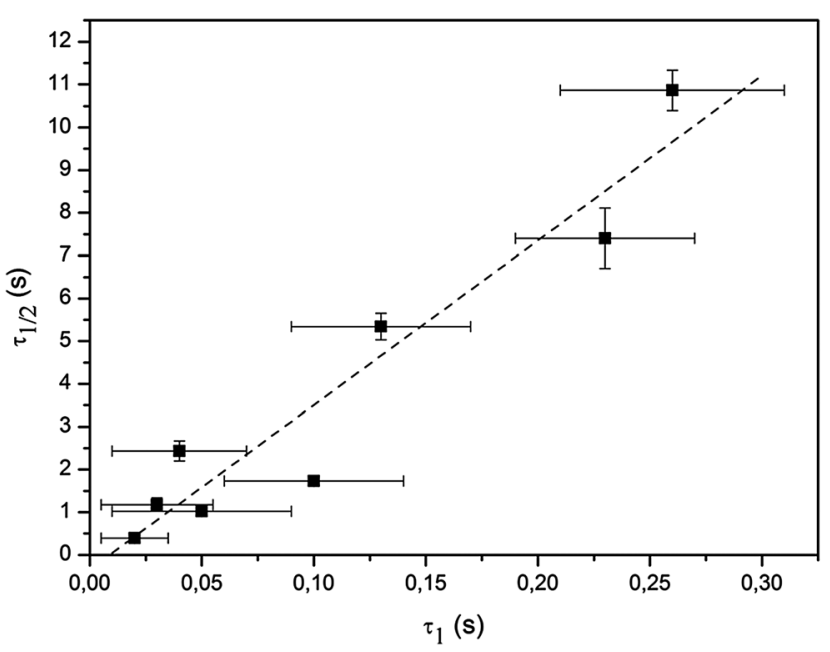

Fig. 5 Plot of $\tau_{1}$ against $\tau_{1 / 2}$ reveals that both timescales are significantly correlated. A linear fit yields $\tau_{1 / 2}=(38.5 \pm 4.8) \times \tau_{1}+(0.3 \pm 0.7) \mathrm{s}$. Obviously, the intersection point with the axis is very close to the origin and suggests the ansatz of a proportionality of both timescales $\tau_{1}=k \tau_{1 / 2}$ with $k=38.5 \pm 4.8$. Data points were obtained from the analysis of native as well as ATP depleted cells.

\section{Discussion}

The two experimentally observed types of fast shape and slow orientational relaxation occur on clearly separated time scales. Sackmann and coworkers were among the first reporting the relaxation of red cells on two significantly different time scales. ${ }^{2}$ They deformed red cells in a high-frequency electric field and observed the response curve for cell elongation obtained after a field jump. A fit with two exponentials yields time constants of $0.1 \mathrm{~s}$ and $1 \mathrm{~s}$, respectively. They explain their observation using a linear Voigt-Maxwell model with two springs and two dissipating dashpots which represent cell membrane and cytoskeleton elasticity as well as the membrane and cytosol viscosity.

Several groups have observed relaxation of RBCs in micropipette aspiration experiments. ${ }^{4,7,44}$ However, typical timescales were of the order of several $100 \mathrm{~ms}$. Because the timescale of the shape relaxation in our experiments is of the same order of magnitude we discuss it within the framework of these seminal works.

\section{Relaxation of shape $\tau_{1}$}

During the faster relaxation red cells transit from a parachute to a cup-shaped stomatocyte. This means that the strongly bent concave rear as well as the (less strongly bent) front dimple relax. Two different contributions to the elastic energies as well as two different dissipations must be considered. ${ }^{4}$

On the one hand, the relaxation reduces the bending energy stored in the cell membrane. On the other hand, a change of the Gaussian curvature in a region of the membrane induces a change in the shear strain of the cytoskeleton. The elastic energy is opposed by the viscous dissipation in both the membrane and the adjacent aqueous phases (cytosol and external fluid). From the balance of bending (with bending rigidity $\kappa$ ) and membrane and bulk dissipation coefficients $\eta_{\mathrm{m}}$ and $\eta$, respectively, Evans derived a characteristic folding (bending) time of ${ }^{4}$

$$
\tau_{\mathrm{b}}=\frac{\nu+\eta R / c^{2}}{\kappa}
$$

where $R$ is the typical radius of the red cell, $c$ the curvature of the fold at the rear and $\nu$ a small and negligible coefficient representing membrane dissipation for bending. Using $R \approx 1 / \mathrm{c} \approx$ $3.3 \mu \mathrm{m}, \kappa=2 \times 10^{-19} \mathrm{~J}$ and $\eta=10^{-3} \mathrm{~Pa}$ yields $\tau_{\mathrm{b}} \approx 180 \mathrm{~ms}$ which nicely fits to the measured range of the experimental values. In micropipette expulsion experiments a typical folding time of $\sim 0.3 \mathrm{~s}$ was measured. ${ }^{45}$ The characteristic time for shear deformation is given by

$$
\tau_{\mathrm{s}}=\frac{\eta_{\mathrm{m}}+\eta R}{\mu} \approx \frac{\eta_{\mathrm{m}}}{\mu} .
$$

Again exploiting micropipette experiments it could be proven that membrane viscosity dominates shear relaxation and the contribution of bulk viscosity is two orders of magnitudes smaller. ${ }^{4}$ Using $\mu=6 \times 10^{-6} \mathrm{~N} \mathrm{~m}^{-1}$ and $\eta_{\mathrm{m}}=10^{-6} \mathrm{~N} \mathrm{~s} \mathrm{~m}^{-1}$ (ref. 7) yields $\tau_{\mathrm{s}}=125 \mathrm{~ms}$, again in the same range as our experiments.

Since the estimation of shear and bending mechanisms yields relaxation times of the same order of magnitude one might ask which contribution is dominant for the elastic energy using a simplified model. We may use the model of a plane circular membrane that is deformed into a half-sphere and estimate the shear strain energy by

$$
E_{0}=\frac{\mu S\left\langle u^{2}\right\rangle}{2} \approx 0.5 \times 10^{-16} \mathrm{~J}
$$

with the surface area of the sphere equal to the area of a red blood cell $S=1 \times 10^{-10} \mathrm{~m}^{2}$ and a mean shear strain $\left\langle u^{2}\right\rangle=0.17$ (see also ref. 46). The bending energy of a half-sphere is

$$
E_{\mathrm{B}}=4 \pi \kappa \approx 2.4 \times 10^{-18} \mathrm{~J}
$$

which is much smaller than $E_{0}$ and makes it plausible that $\tau_{1}$ only depends on the shear elastic and membrane dissipative properties of the red blood cell membrane; therefore $\tau_{1} \approx \tau_{\mathrm{s}}$.

\section{Relaxation of orientation $\tau_{1 / 2}$}

An orientational relaxation of red blood cells, first observed by Fischer, ${ }^{10}$ has been associated by Skotheim and Secomb ${ }^{46}$ with tank-treading motion in simple shear flow. For tank-treading, the angular velocity is determined by the equilibrium of driving force (external shear flow) and internal dissipation in the membrane. They extended the Keller-Skalak model and included the effect of an elastic membrane ${ }^{47}$ and assumed the cytoskeleton to rotate while the orientation angle and overall shape were virtually conserved in tank treading. According to the relative position of the cytoskeleton with respect to the orientation of the shape the elastic energy of the cytoskeleton varies. They therefore conclude that the elastic energy $E$ of the cytoskeleton is a periodic function of the relative angle $\varphi$ between the shape and the cytoskeleton with a periodicity of $\pi$, and suggest the simple ansatz $E=E_{0} \sin ^{2} \varphi$. The maximal amplitude $E_{0}$, that is the maximum energy stored in the shear strained cell 
membrane, can be estimated by the maximum of the orientational rotation velocity $\omega_{\max }$ and the viscosity $\eta^{\prime}=\eta_{\mathrm{m}} / R$,

$$
E_{0}=V \eta^{\prime} f_{1} \omega_{\max }
$$

with the red cell's volume $V=90 \mu \mathrm{m}^{3}$ (ref. 48) and a geometry factor $f_{1} \approx 15 / 4$ describing the degree of deformation. ${ }^{46}$

However, in the case of tank treading the driving force is the external shear flow, while in our experiments rotation is driven by the elastic energy stored in the cytoskeleton and $\omega_{\max }$ denotes the maximum of the angular rotation.

Our experiments suggest that there is a linear dependence of the tilt angle $\varphi$ on time $t$ as shown in Fig. 3b. Assuming the dissipating forces to be constant at constant angular velocity $\omega=$ $\mathrm{d} \varphi / \mathrm{d} t$ this implies a constant driving momentum to balance. Only when the relative tilt angle vanishes (has reached its equilibrium value) the moment abruptly becomes zero. Instead of the above theoretically assumed smooth dependence $E \sim \sin ^{2} \varphi$ our experiments suggest a sharper triangular oscillation with the same periodicity $\pi$.

A consequence of the periodic dependence of the elastic energy of the cytoskeleton network on $\varphi$ is that the continuous tank treading motion of red blood cells is affected. This leads to a swinging motion superposed on top of the tank treading motion and was first observed by Abkarian et al. for red blood cells. ${ }^{49}$ Their data show typical triangular characteristics (zigzag) of the swinging angle with time which is in excellent agreement with the sharp contour found in our experiments.

We may estimate the value for $\omega_{\max }$ from eqn (5) using the formula for shear elastic energy estimated in eqn (3), with a mean shear strain $u=0.2$ as estimated by Skotheim and Secomb for tank-treading RBCs. ${ }^{46}$ This results in a relaxation time

$$
\tau_{1 / 2}=\frac{\eta^{\prime}}{\mu} \frac{V f_{1} \pi}{2 S\left\langle u^{2}\right\rangle} \approx 6.7 \mathrm{~s}
$$

which nicely corresponds to the value of our experiments. For reasons of this consistency and the fact that the measurements by Fischer ${ }^{10}$ yield values in the same range of 5-20 s, we infer that the proposed mechanism explains our observations for red cell rotation.

\section{Correlation of the relaxation timescales $\tau_{1}$ and $\tau_{1 / 2}$}

Furthermore, we have plotted the correlation of the two timescales of single red blood cells as observed in the experiments and found that with increasing or decreasing $\tau_{1}$ the value of $\tau_{1 / 2}$ increases or decreases accordingly as shown in Fig. 5. The plot suggests a linear dependence of both relaxation times with a very small value of the ordinate. Because $\tau_{1} \sim \eta_{\mathrm{m}} / \mu$ we may conclude that the contribution of bending to the relaxation time $\tau_{1}$ is small and therefore $\tau_{1} \approx \tau_{\mathrm{s}}$. Hence, assuming a line through the origin which is justified by our experiments, both relaxation times are proportional: $\tau_{1 / 2}=$ $(36.4 \pm 4.7) \tau_{1}$. Using eqn (2) and eqn (6) allows us to relate the two characteristic time scales theoretically by

$$
\frac{\tau_{1 / 2}}{\tau_{1}}=\frac{\pi V f_{1}}{2 R\left\langle u^{2}\right\rangle S} \approx 40
$$

which is in excellent agreement with the experimental value obtained from Fig. 5.

\section{Variation of RBC properties using ATP}

ATP is known to bind to the cytoskeleton of the cell ${ }^{50}$ and modify the elastic moduli of the cell. To study the effect of ATP, we metabolically depleted the ATP concentration. According to our model, the shear elasticity $\mu$ is proportional to the inverse of the rotational relaxation time $\tau_{1 / 2}$. In Fig. 6 , we plot $1 / \tau_{1 / 2}$ versus ATP concentration in the $\mathrm{RBC}$, normalized by the fastest relaxation time $\tau_{1 / 2}^{\mathrm{e}}=0.29 \mathrm{~s}$ serving as an estimation of a RBC with very low ATP level.

One can clearly see that shear elasticity decreases with increasing ATP levels until it reaches a saturation level at an ATP content of $\sim 0.4 \mathrm{mmol}^{-1}$, which is about $30 \%{ }^{17}$ of the content of fresh red cells. We therefore propose a simple ATP adsorption model for red cell softening: the red cell cytoskeleton contains ATP binding sites where ATP from the cell plasma can bind and unbind, according to a chemical equilibrium equation:

$$
[\mathrm{ATP}]+[\mathrm{B}] \leftrightarrow[\mathrm{ATPB}]
$$

The occupation probability $n_{\mathrm{a}}$ can then be calculated from ATP concentration in the cell plasma $n_{\mathrm{ATP}}$ and absorption energy $\varepsilon$ (standard Gibbs energy change; in units of $\left.k_{\mathrm{B}} T\right)^{1}$ by standard thermodynamics:

$$
n_{\mathrm{a}}=\frac{n_{\mathrm{ATP}} \mathrm{e}^{\varepsilon}}{1+n_{\mathrm{ATP}} \mathrm{e}^{\varepsilon}}
$$

Assuming that bound ATP initiates a process that softens the cytoskeleton, the shear elasticity is considered to depend on the ratio of free binding sites, $n_{0}=\left(1-n_{\mathrm{a}}\right)$.

When we fit $n_{0}$ to our normalized relaxation times $1 / \tau_{1 / 2}$ with $\varepsilon$ as a single fit parameter as shown is Fig. 6, we find for the best fit a value of $\varepsilon=10$ in units of $k_{\mathrm{B}} T$, which is reasonable. ${ }^{1}$

To relate ATP concentration to the elastic moduli, several models of ATP-induced cytoskeleton softening have been proposed.

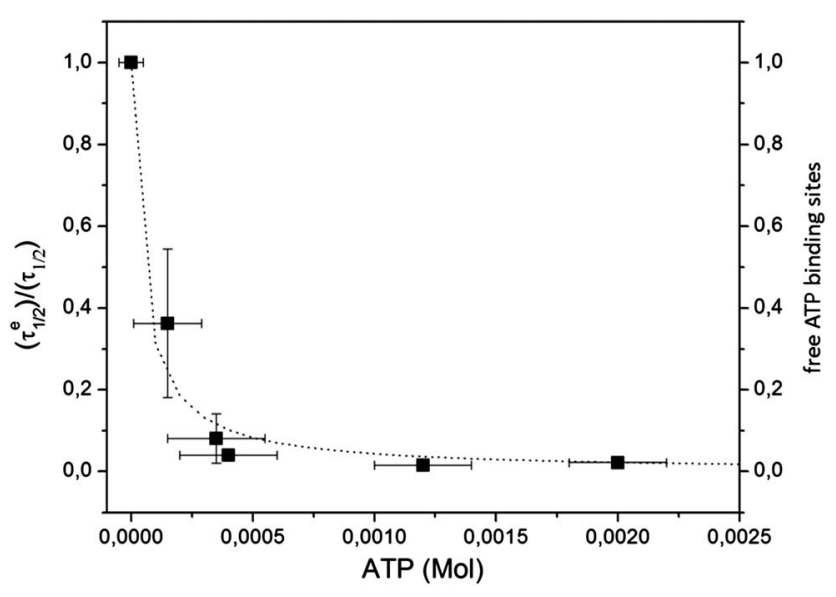

Fig. 6 Dependence of $\tau_{1 / 2}$ mean values on ATP concentration (left ordinate). Values are normalized by $\tau_{1 / 2}$ of the fastest relaxing echinocyte measured. The dotted line is a fit of our model of ATP-binding equilibrium, with the binding energy $\varepsilon$ as a single fit parameter (right ordinate: number of non-occupied binding sites). 
Gov and Safran suggest that softening is caused by ATPinduced phosphorylation of the protein-IV.1, which triggers the disconnection of one spectrin filament from the actin node. ${ }^{1,42}$ In ref. 1 the adsorption energy of ATP to the spectrin-actin site is estimated by $\varepsilon \sim 9$ (in units of $k_{\mathrm{B}} T$ ) and related to spectrin connectivity and shear modulus, which is in good agreement with our result $\varepsilon=10$. They propose the shear elasticity to be proportional to the ratio of free binding sites $n_{0}$.

In a dynamic coarse grain model Zhang and Brown suggest that ATP-induced changes may also soften each spectrin link and therefore contribute to red cell softening. Hence, even when all ATP binding sides are occupied, the network does not fully dissolve and shape memory could be preserved. ${ }^{51}$ To account for this observation, Yoon et al. proposed a model with two types of bonds, ATP-influenced transient bonds and permanent bonds which prevent total fluidization of the network. ${ }^{24,52}$

Recently, Manno et al. ${ }^{53}$ investigated the effects of glycation (non-enzymatic glycosylation) of spectrin filaments in red blood cells. They observed that glycation leads to a stiffening of the membrane and provided evidence that phosphatidylserine, which is actively transported to the inner membrane leaflet by ATPdriven proteins, prevents glycation at physiological ATP concentrations. This also nicely coincides with the observed increase of softening mediated by ATP depletion in our experiments.

Additionally, spectrin filaments can not only dissociate from the actin node but also from their mid-point attachment to the plasma membrane between the actin nodes. This dissociation on the one hand increases the re-association time of spectrin-actin dissociations and therefore further weakens the cytoskeleton shear elasticity. On the other hand, it increases the elastic modulus by increasing the degrees of freedom of the spectrin which acts as an entropic spring. The net effect is a stiffening of the membrane. ${ }^{1,54}$

ATP-induced breaking of cytoskeleton filaments as proposed by Gov and Safran ${ }^{1}$ would facilitate loss of shape memory, by long-scale rearrangement of the cytoskeleton structure.

In ref. 1 and 54 it was argued that network softening occurs if the spectrin filaments are cut at both ends. ATP can induce such doubly cut dissociations that cause a decrease in shear modulus. However, a spectrin filament is most likely to reassociate at the same node where it was dissociating, thus conserving shape memory. For a reorganization or fluidization the spectrin filament has to reassociate at a different node and therefore needs to overcome an energy barrier. External shear stress may provide this additional energy. ${ }^{9,27}$ Fischer ${ }^{10}$ showed that at shear rates of $\dot{\gamma} \sim 100 \mathrm{~s}^{-1}$ the shape memory was found to be stable for $>80$ min. Since in our experiments the red cells were subjected to shear flow for less than $10 \mathrm{~s}$ in the microchannel, a loss of shape memory was not to be expected, and indeed was not observed, as all cells regained their biconcave discocyte shape after full relaxation.

The energy barrier may also be overcome by the thermal energy. We incubated the red blood cells for a maximal period of $60 \mathrm{~min}$ in the repletion experiments, and metabolic depletion was done for maximally 72 hours. Within this time span no significant shape deviation from the resting discocyte was found, again demonstrating that shape memory was not affected.
It is known that ATP levels as well as activity of ATP dependent enzymes between cells of a single donor largely scatter, probably due to cell ageing. ${ }^{55,56}$ The correlation of relaxation time and ATP level gives a reasonable explanation for the large scattering of the values for $\tau_{1 / 2}$ and $\tau_{1}$, which have been also reported by other groups. ${ }^{5,10}$

The significantly enhanced scattering at higher ATP levels as compared to a lower scattering for ATP depleted cells can be understood by considering simple enzyme kinetics. At high ATP levels enzyme activity is rate limiting and the comparatively large natural variation of enzyme activity results in large variation of network connectivity and thus variation of $\tau_{1 / 2}$. At low ATP levels the substrate (ATP) concentration is rate limiting and because ATP concentrations vary to a lesser extent variation of values measured is reduced.

However, clearly more experiments are necessary to reveal the complex mechanisms of ATP dependent processes in red cell deformation dynamics.

\section{Conclusion}

We developed a microfluidic device which opens new possibilities for studying the relaxation of strongly deformed erythrocytes (such as parachute shaped cells) into the discoid ground state. This could mimic physiological situations such as the passage of erythrocytes from narrow capillaries into the large blood vessels. The relaxation consists of a rapid transition from parachute-like into (often) distorted stomatocyte-like shapes (in about $200 \mathrm{~ms}$ ) which is followed by a slower relaxation of the stomatocyte-like shape into the discoid ground state associated with a rotation of the cell.

Using ATP we were able to correlate the fast relaxation time $\tau_{1}$ with the rotational relaxation time $\tau_{1 / 2}$ and found a linear relationship that indicates that both time scales depend on the cytoskeleton properties. Both the values of the relaxation times as well as their linear relationship could be understood in terms of a viscoelastic theoretical model.

During aging in vivo and in vitro RBCs undergo several biochemical changes, such as ATP depletion, which strongly affect the properties of the cytoskeleton. ${ }^{56,57}$ ATP-depletion results in the shortening of the short and long relaxation times by an order of magnitude, and the changes are completely reversible after increasing the ATP content to the normal concentration, suggesting that the transition from the parachute to the discocyte shape is actively controlled by the ATP-mediated reorganisation of the cytoskeleton.

While shape changes of giant vesicles are controlled solely by the area to volume ratio and spontaneous curvature, those of erythrocytes are controlled by the coupling of the spectrin-actin network to the membrane. Important consequences of this coupling between the two sub-shells are complex fluctuation spectra $^{58}$ and the generation of local gradients of lateral tension for non-spherical shells. ${ }^{59} \mathrm{In}$ order to maintain this coupling, any deformation of the composite shell from its equilibrium shape is associated with a local gradient of the lateral tension of the cytoskeleton, causing a change in network density and network elasticity. ${ }^{9,60}$ Such a gradient is compressive for concave deflection (that is if the shell is deformed in the direction of the cytoskeleton) since the cytoskeleton is expanded and tries to restore the equilibrium. 
One consequence of the spatial variation of the tension and the associated density of the spectrin network is the non-isotropic distribution of band III proteins mediating the spectrin bilayer coupling. ${ }^{61}$ In order to stabilize a cup-shape the mesh size and the density of band III are increased at the convex and decreased at the concave side. Therefore, any shape change induced by external forces is associated with a restructuring of the cytoskeleton. The time of restructuring is determined by the dynamics of the binding-unbinding equilibrium of the spectrin and possibly the diffusion of the protein band III.

We can understand the reorganisation of the cytoskeleton in terms of the concept of homeostasis. The transition from the discocyte to the parachute enforced by the hydrodynamic shear generates a large elastic stress. In order to minimize stress induced damage of the composite shell, the cell responds actively (that is under ATP consumption) by reorganisation of the cytoskeleton in such a way that the elastic stress is reduced. The restructuring during the relaxation of the cell into the ground state (discocyte) can go over several intermediate metastable states, such as stomatocyte-like shapes, and each transition exhibits a different relaxation time.

Generally, ATP plays an important physiological role in the cardiovascular system. For instance, ATP is released from deformed red blood cells $\mathrm{s}^{21,23}$ and upon increased workload of the heart ATP is released from the myocardium. ${ }^{62}$ Besides the well known vasodilating effect of ATP, ${ }^{63}$ our experiments suggest that increased cardiac plasma ATP levels also enhance blood flow efficiency due to the demonstrated ATP dependent regulation of shear elasticity. ${ }^{36,64}$ Because different types of red blood cell motion such as tank-treading, tumbling and swinging critically depend on the elastic properties of the composed cell's membrane, regulation by ATP allows to regulate cell function as well. ${ }^{65-67}$

The presented results are also highly interesting in a more general context, as the spectrin network is attached to most cellular membrane systems such as Golgi membranes, intracellular vesicles in cerebellar neurons and on plasma membranes. Sensitivity of proteins to mechanical load is likely to play a key role not only in regulation of deformability but also in various mechano-signaling processes ${ }^{68,69}$ Recently, it has been proposed that shear induced unfolding of spectrin exposes hidden binding sites which could act as mechano-sensors. ${ }^{25}$ Thus, ATP regulated cytoskeleton connectivity and relaxation offers an alternative smart way to regulate such mechano-signaling pathways.

\section{Acknowledgements}

TF acknowledge support of the German Excellence Initiative via NIM, the Centre for Nanoscience CeNS and the German Research Society DFG via the priority programs SPP1164 and SPP1253. TF particularly likes to thank Achim Wixforth for continuously supporting this work.

\section{References}

1 N. S. Gov and S. A. Safran, Red blood cell membrane fluctuations and shape controlled by ATP-induced cytoskeletal defects, Biophys. $J ., 2005, \mathbf{8 8}, 1859-1874$

2 H. Engelhardt, H. Gaub and E. Sackmann, Viscoelastic properties of erythrocyte membranes in high-frequency electric field, Nature, 1984, 307, 378-380.
3 H. Engelhardt and E. Sackmann, On the measurement of shear elastic moduli and viscosities of erythrocyte plasma membranes by transient deformation in high frequency electric fields, Biophys. J., 1988, 54(3), 495-508.

4 E. A. Evans, Structure and deformation properties of red blood cells: concepts and quantitative methods, Methods Enzymol., 1989, 173, 3.

5 P. J. H. Bronkhorst, et al., A new method to study shape recovery of red blood cells using multiple optical trapping, Biophys. J., 1995, 69, 1666-1673.

6 K. E. Bremmell, A. Evans and C. A. Presidge, Deformation and nanorheology of red blood cells: an AFM investigation, Colloids Surf., B, 2006, 50, 43-48.

7 R. E. Waugh and R. M. Hochmuth, Erythrocyte membrane elasticity and viscosity, Аnпи. Rev. Physiol., 1987, 49, 209-219.

$8 \mathrm{~S}$. Tuvia, et al., Cell membrane fluctuations are regulated by medium macroviscosity: evidence for a metabolic driving force, Proc. Natl. Acad. Sci. U. S. A., 1997, 94(10), 5045.

9 J. C.-M. Lee and D. E. Discher, Deformation-enhanced fluctuations in the red cell skeleton with theoretical relations to elasticity, connectivity, and spectrin unfolding, Biophys. J., 2001, 81, 3178-3192.

10 T. M. Fischer, Shape memory of human red blood cells, Biophys. J., 2004, 86, 3304-3313.

11 A. Nans, N. Mohandas and D. L. Stokes, Native ultrastructure of the red cell cytoskeleton by cryo-electron tomography, Biophys. J., 2011, 101(10), 2341-2350

12 S. Manno, Y. Takakuwa and N. Mohandas, Modulation of erythrocyte membrane mechanical function by protein 4.1 phosphorylation, J. Biol. Chem., 2005, 280(9), 7581.

13 T. Betz, et al., ATP-dependent mechanics of red blood cells, Proc. Natl. Acad. Sci. U. S. A., 2009, 106(36), 15320.

$14 \mathrm{~S}$. Manno, et al., Modulation of erythrocyte membrane mechanical function by-spectrin phosphorylation and dephosphorylation, $J$. Biol. Chem., 1995, 270(10), 5659.

15 E. Sackmann, Membrane bending energy concept of vesicle-and cellshapes and shape-transitions, FEBS Lett., 1994, 346(1), 3-16.

16 Y. Park, et al., Metabolic remodeling of the human red blood cell membrane, Proc. Natl. Acad. Sci. U. S. A., 2010, 107(4), 1289-1294.

17 C. Feo and N. Mohandas, Clarification of the role of ATP in red-cell morphology and function, Nature, 1977, 265, 166-168.

$18 \mathrm{~S}$. Tuvia, et al., Mechanical fluctuations of the membrane-skeleton are dependent on F-actin ATPase in human erythrocytes, J. Cell Biol., 1998, 141(7), 1551.

19 R. I. Weed, P. L. LaCelle and E. W. Merrill, Metabolic dependence of red cell deformability, J. Clin. Invest., 1969, 48(5), 795.

20 P. Boivin, Role of the phosphorylation of red blood cell membrane proteins, Biochem. J., 1988, 256(3), 689.

21 J. Wan, A. M. Forsyth and H. A. Stone, Red blood cell dynamics: from cell deformation to ATP release, Integr. Biol., 2011, 3(10), 972-981.

22 A. K. Price, et al., Deformation-induced release of ATP from erythrocytes in a poly(dimethylsiloxane)-based microchip with channels that mimic resistance vessels, Anal. Chem., 2004, 76, 48494855 .

23 C. Wang, X. Wang and P. Ye, The Transport and Deformation of Blood Cells in Micro-Channel, in 3rd IEEE Int. Conf. on Nanol Micro Engineered and Molecular Systems, Sanya, China, 2008.

$24 \mathrm{X}$. An, et al., Shear-response of the spectrin dimer-tetramer equilibrium in the red blood cell membrane, J. Biol. Chem., 2002, 277(35), 31796.

25 C. P. Johnson, et al., Forced unfolding of proteins within cells, Science, 2007, 317(5838), 663.

26 D. E. Discher, N. Mohandas and E. A. Evans, Molecular maps of red cell deformation: hidden elasticity and in situ connectivity, Science, 1994, 266(5187), 1032.

$27 \mathrm{~J}$. Li, et al., Cytoskeletal dynamics of human erythrocyte, Proc. Natl. Acad. Sci. U. S. A., 2007, 104(12), 4937-4942.

28 E. Sackmann, Thermo-elasticity and adhesion as regulators of cell membrane architecture and function, J. Phys.: Condens. Matter, 2006, 18, R785-R825.

29 R. E. Waugh, Effects of inherited membrane abnormalities on the viscoelastic properties of erythrocyte membrane, Biophys. J., 1987, 51, 363-369.

30 N. Mohandas and E. Evans, Mechanical properties of red cell membrane in relation to molecular structure and genetic defects, Annu. Rev. Biophys. Biomol. Struct., 1994, 23, 787-818. 
31 N. Mohandas and P. G. Gallagher, Red cell membrane: past, present, and future, Blood, 2008, 112(10), 3939-3948.

32 N. S. Gov, Less is more: removing membrane attachments stiffens the RBC cytoskeleton, New J. Phys., 2007, 9, 429.

33 M. C. Murray and M. E. Perkins, Phosphorylation of erythrocyte membrane and cytoskeleton proteins in cells infected with plasmodium falciparum, Mol. Biochem. Parasitol., 1989, 34(3), 229-236.

34 H. Noguchi, et al., Dynamics of fluid vesicles in flow through structured microchannels, Europhys. Lett., 2010, 89, 28002.

35 G. Tomaiuolo, et al., Red blood cell deformation in microconfined flow, Soft Matter, 2009, 5(19), 3736-3740.

36 H. Noguchi and G. Gompper, Shape transitions of fluid vesicles and red blood cells in capillary flows, Proc. Natl. Acad. Sci. U. S. A., 2005, 102(40), 14159-14162.

37 M. Nakao, et al., Adenosine triphosphate and shape of erythrocytes, J. Biochem., 1961, 49(6), 487.

38 D. C. Duffy, et al., Rapid prototyping of microfluidic systems in poly (dimethylsiloxane), Anal. Chem., 1998, 70(23), 4974-4984.

39 M. Bessis, Living Blood Cells and their Ultrastructure, SpringerVerlag, Berlin, Heidelberg, New York, 1973.

$40 \mathrm{H}$. L. Goldsmith and J. Marlow, Flow behaviour of erythrocytes. I. Rotation and deformation in dilute suspensions, Proc. $R$. Soc. London, Ser. B, 1972, 182(1068), 351.

$41 \mathrm{M}$. Abkarian and A. Viallant, Vesicles and red blood cells in shear flow, Soft Matter, 2008, 4, 653-657.

42 N. S. Gov, Active elastic network: cytoskeleton of the red blood cell, Phys. Rev. E: Stat., Nonlinear, Soft Matter Phys., 2007, 75(1), 011921.

$43 \mathrm{G}$. Brecher and M. Bessis, Present status of spiculed red cells and their relationship to the discocyte-echinocyte transformation: a critical review, Blood, 1972, 40(3), 333-344.

$44 \mathrm{~S}$. Chien, et al., Theoretical and experimental studies on viscoelastic properties of erythrocyte membrane, Biophys. J., 1978, 24(2), 463487.

45 E. Evans and A. Leung, Adhesivity and rigidity of erythrocyte membrane in relation to wheat germ agglutinin binding, J. Cell Biol., 1984, 98, 1201-1208.

46 J. M. Skotheim and T. W. Secomb, Red blood cells and other nonspherical capsules in shear flow: oscillatory dynamics and the tank-treading-to-tumbling-transition, Phys. Rev. Lett., 2007, 98(078301), 1-4.

47 S. R. Keller and R. Skalak, Motion of a tank-treading ellipsoidal particle in a shear flow, J. Fluid Mech., 2006, 120, 27-47.

48 S. Chien, Red cell deformability and its relevance to blood flow, Аnпи. Rev. Physiol., 1987, 49, 177-192.

49 M. Abkarian, M. Faivre and A. Viallat, Swinging of red blood cells under shear flow, Phys. Rev. Lett., 2007, 98, 188302.

$50 \mathrm{H}$. Chu, et al., Identification of cytoskeletal elements enclosing the ATP pools that fuel human red blood cell membrane cation pumps, Proc. Natl. Acad. Sci. U. S. A., 2012, 12794.
51 R. Zhang and F. L. H. Brown, Cytoskeleton mediated effective elastic properties of model red blood cell membranes, J. Chem. Phys., 2008, 129, 065101.

52 Y. Z. Yoon, et al., Red blood cell dynamics: from spontaneous fluctuations to non-linear response, Soft Matter, 2011, 7, 2042-2051.

53 S. Manno, N. Mohandas and Y. Takakuwa, ATP-dependent mechanism protects spectrin against glycation in human erythrocytes, J. Biol. Chem., 2010, 285(44), 33923.

54 N. S. Gov, Less is more: removing membrane attachments stiffens the RBC cytoskeleton, New J. Phys., 2007, 9(11), 429.

55 F. I. Ataullakhanov and V. M. Vitvitsky, What determines the intracellular ATP concentration, Biosci. Rep., 2002, 22(5), 501-511.

$56 \mathrm{H}$. K. Jindal, et al., Specific loss of protein kinase activities in senescent erythrocytes, Blood, 1996, 88(4), 1479.

57 M. Girasole, et al., The how, when, and why of the aging signals appearing on the human erythrocyte membrane: an atomic force microscopy study of surface roughness, Nanomedicine: Nanotechnology, Biology and Medicine, 2010, 6(6), 760-768.

58 T. Auth, S. A. Safran and N. S. Gov, Fluctuations of coupled fluid and solid membranes with application to red blood cells, Phys. Rev. E: Stat., Nonlinear, Soft Matter Phys., 2007, 76(5), 051910.

59 R. Mukhopadhyay, H. W. G. Lim and M. Wortis, Echinocyte shapes: bending, stretching, and shear determine spicule shape and spacing, Biophys. J., 2002, 82, 1756-1772.

60 J. C. M. Lee, D. T. Wong and D. E. Discher, Direct measures of large, anisotropic strains in deformation of the erythrocyte cytoskeleton, Biophys. J., 1999, 77(2), 853-864.

61 E. Sackmann, Thermo-elasticity and adhesion as regulators of cell membrane architecture and function, J. Phys.: Condens. Matter, 2006, 18, R785.

$62 \mathrm{~K}$. Mubagwa, K. Mullane and W. Flameng, Role of adenosine in the heart and circulation, Cardiovasc. Res., 1996, 32(5), 797.

63 R. S. Sprague, et al., ATP: the red blood cell link to NO and local control of the pulmonary circulation, Am. J. Physiol.: Heart Circ. Physiol., 1996, 271(6), H2717.

64 V. Vitkova, et al., Micro-macro link in rheology of erythrocyte and vesicle suspensions, Biophys. J., 2008, 95(6), L33-L35.

65 M. L. Ellsworth, et al., Erythrocytes: oxygen sensors and modulators of vascular tone, Physiology, 2009, 24(2), 107.

66 B. Kaoui, G. Biros and C. Misbah, Why do red blood cells have asymmetric shapes even in a symmetric flow?, Phys. Rev. Lett., 2009, 103(18), 188101.

67 C. Wang and A. S. Popel, Effect of red blood cell shape on oxygen transport in capillaries, Math. Biosci., 1993, 116, 89-110.

68 A. J. Engler, et al., Matrix elasticity directs stem cell lineage specification, Cell, 2006, 126(4), 677-689.

69 B. Geiger, J. P. Spatz and A. D. Bershadsky, Environmental sensing through focal adhesions, Nat. Rev. Mol. Cell Biol., 2009, 10(1), 21-33. 